\title{
Effect of Conditioning Downforce and Pad Break-In Time on Pad Surface Micro-Texture
}

\author{
Jeffrey McAllister $^{1 *}$, Calliandra Stuffle ${ }^{1}$, Yasa Sampurno ${ }^{1,2}$, Dale Hetherington ${ }^{3}$, Jon Sierra \\ Suarez ${ }^{3}$, Leonard Borucki ${ }^{2}$ and Ara Philipossian ${ }^{1,2}$ \\ ${ }^{1}$ Department of Chemical and Environmental Engineering, \\ University of Arizona, Tucson, Arizona, 85721 USA \\ ${ }^{2}$ Araca Inc, Tucson, Arizona, 85718 USA \\ ${ }^{3}$ Sandia National Laboratories, Albuquerque, New Mexico, 87116 USA
}

The effect of conditioning downforce during pad break-in, and its impact on the evolution of pad surface micro-texture was investigated. Two different conditioning downforces were used to break-in pads. Pad samples were extracted after certain break-in times and analyzed for their topography and contact area using confocal microscopy. Results showed that the pad-wafer contact area and contact density decreased with conditioning downforce. Break-in at the higher conditioning downforce helped in reaching faster stabilized values of the analyzed micro-texture parameters. The evolution of these parameters was different for the two different downforces, however as break-in time increased past 30 minutes, mean summit height and mean summit curvature began to approach approximately the same value. These results indicated that, for the particular disc used in this study, a change in the magnitude of downforce during pad break-in caused a change in break-in time and stable values for some micro-texture parameters. However, a 
change in the magnitude of downforce during break-in only resulted in a change in break-in time for other micro-texture parameters. The work underscored the need for conducting such tests with different conditioners in order to develop a better understanding of the role downforce plays on the evolution of the micro-texture.

* Corresponding author: Jeffrey McAllister

*Email: jemcalli@email.arizona.edu 


\section{Introduction}

Chemical mechanical planarization (CMP) is a process that has been successfully used to achieve global and local planarization across the wafer surface in integrated circuit (IC) manufacturing since the 1990s. CMP involves a rotating wafer, mounted face down in a carrier, being placed into contact with a rotating polishing pad. Slurry containing abrasive particles and chemical constituent is injected, typically, near the center of the pad. The grooves and pores of the pad coupled with the kinematics of the polishing process accommodate the slurry transport to the pad-wafer interface where the pad asperities, the wafer, and the slurry with its abrasive particles come into contact. The combination of these three-body contacts among pad, slurry, and wafer leads to removal of material across the entire wafer surface ${ }^{(1)}$ Concurrently, the pad surface micro-texture becomes degraded by abrasive wear, plastic deformation on the pad surface, and build-up of polishing byproducts on the pad surface. In order to maintain a steady CMP process, a pad conditioning process is necessary to counteract the degradation of the pad surface micro-texture. ${ }^{(2)} \mathrm{A}$ diamond or CVDcoated conditioning disc rotates against the pad surface in a similar manner to the wafer being polished. At the current state-of-the-art, a newly installed polishing pad has to be sufficiently broken-in to establish an initial pad surface micro-texture to achieve a consistent material removal rate from one wafer to another. During pad break-in, ultra-pure water (UPW) is injected onto the pad surface and a conditioning disc is employed to make the pad surface reach (and remain at) a desired surface micro-texture (i.e. the combination of asperity heights, densities, area, and radii of curvature). Two conditioning factors that affect pad surface micro-texture are conditioning downforce and pad break-in time. ${ }^{(3,4)}$ In typical high volume manufacturing processes, pad breakin times range from approximately 20 to 45 minutes, and a shorter break-in time is sought after in order to increase polisher availability and throughput. It is therefore important to understand the 
behavior of the surface micro-texture of the pad during the break-in process and how this process is affected by conditioning downforce.

Laser scanning confocal microscopy has been successfully implemented and proven to be very effective in studying the pad surface micro-texture. This technique has been used to gain a better understanding of how surface micro-texture affects CMP performance. ${ }^{(4-10)}$ Sun et al. studied the effect of conditioning downforce on copper CMP pad topography and found that the pad surface became more abrupt as conditioning downforce decreased. ${ }^{(4)}$ Sampurno et al. studied the correlation between pad topography and tungsten removal rate, and found a direct correlation between pad surface abruptness and removal rate. ${ }^{(5)}$ Mu et al. also studied the relationship between pad topography and tungsten removal rate, yet they reported that lower values of pad surface abruptness caused higher removal rates. ${ }^{(6)}$ Separately, Liao et al. found that a pad conditioned at a higher downforce resulted in a higher oxide removal rate due to a dramatic decrease in pad surface contact area, therefore an increase in local contact pressure. ${ }^{(11)}$ These studies provide evidence that the magnitude of conditioning downforce can cause different outcomes to the pad surface microtexture. As such, there is a need to further investigate the relationship between conditioning downforce and its effect on the micro-texture during oxide CMP. Jeong et al. studied the effect of break-in time on oxide removal rate. ${ }^{(12)}$ However, the micro-texture of the pad surface during the break-in process was not reported. Vasilev et al. found a relationship between pad break-in time, surface roughness, and pad surface height distribution for oxide CMP, using two different CVDcoated diamond discs at $6.6 \mathrm{lb}$. Furthermore, Vasilev et al. used confocal microscopy to find that steady-state pad conditions were reached within 20 to 50 minutes of the pad break-in process for the two different discs being studied. ${ }^{(7)}$ However, no specific relationship between individual pad surface micro-texture parameters (i.e. mean summit height, mean summit curvature, contact 
density, and percentage contact area) was reported. Additionally, the experimental setup used by Vasilev el al. did not involve any form of a pressurized pad wafer contact analysis. Vasilev et al. also recommended that future research on the effect of the conditioning load at a higher downforce would be beneficial in providing clearer insight into the characterization of the oxide CMP process. ${ }^{(7)}$

In this study, two new pads were broken-in by a CVD-coated conditioning disc at two different downforces. During break-in, pad samples were extracted as a function of time and analyzed with confocal microscopy to elucidate the evolution of pad surface micro-texture. Confocal microscopy of the pad samples was conducted using topography mode as well as contact mode. The subsequent image analysis reported mean summit height, summit height distribution, mean summit curvature, contact density, and percentage contact area. The evolution of these pad parameters were compared between the two different conditioning downforces to better understand the effect that conditioning downforce had during break-in. We hope that our present work will help provide a better understanding of individual pad parameter evolution during pad break-in and the effect of conditioning downforce on these surface micro-texture parameters. Such a study is important because previously published works have shown that the pad surface micro-texture has important effects in CMP such as material removal rate, within-wafer-nonuniformity and wafer-level defects. ${ }^{(4-10)}$

\section{Experimental Apparatus and Procedure}

Pad break-in experiments were performed on an Araca APD-800 polisher and tribometer. The system has been described in detail elsewhere. ${ }^{(13)}$ Two IC1000 K-grooved pads with Suba IV sub- 
pad (manufactured by Dow Electronic Materials) having a diameter of $77.5 \mathrm{~cm}$ were broken-in with an Ehwa CVD-coated diamond disc, manufactured by Ehwa Diamond Industrial. One brand new pad was broken-in under a conditioning downforce of $6 \mathrm{lb} f$ while the other was broken-in at $10 \mathrm{lb}$. Both platen and the conditioning disc rotated counter-clockwise at 87 and 60 RPM, respectively. UPW was injected near the pad's center at a flowrate of $250 \mathrm{~mL} / \mathrm{min}$ during breakin. Pad samples were extracted from the center of the wafer track prior to break-in, and after 5, 15, 30 and 60 minutes of the break-in time. Each extracted pad sample was approximately $2 \times 2 \mathrm{~cm}^{2}$.

A Zeiss 880 LSM laser scanning confocal microscope was employed to take topographic images of the surface land areas between two grooves. For each sample, 8 contiguous and non-overlapping images, with an area of $425 \times 425 \mu \mathrm{m}^{2}$ per image, were taken and stitched together to form one image covering a total land area of 3,400 $\times 425 \mu^{2}$. Based on the stitched topography image, a surface height histogram or surface height probability density function (PDF) was constructed. The confocal microscope measures surface heights over a selected area relative to an arbitrary reference plane used by the tool. The height range is divided into equal bins and a histogram is made of the number of times that the surface height falls into each bin. Since the total count in each bin depends on the size of the region measured, it is convenient to normalize the histogram by dividing it by the area under the curve. ${ }^{(8,9)}$ Due to this normalization, the result does not depend on the sampling area and the histogram is then called a PDF. The area under the PDF in any chosen height range gives the probability of finding a point on the surface within that range. Since the reference plane used by the confocal microscope is arbitrary, it is also common to shift the mean of the PDF so that it is at zero. This facilitates the comparison of PDFs from different pad surfaces or different parts of the same surface. ${ }^{(8,9)}$ The summit heights can then be calculated using the surface height mean plane as the baseline. Using this baseline, the mean summit height can be 
determined. The Gaussian curvature of a summit can be calculated by multiplying the two principle curvatures of a summit. These two principle curvatures occur at the values in which curvature is at maximum and minimum. The mean summit curvature is the mean of the calculated Gaussian curvatures. It should be noted that only the peaks that lie in the top $25 \%$ of the surface are used in the analysis of the mean summit height and mean summit curvature. The uppermost summits of the pad topography are what is most important in CMP. It is for this reason that the top $25 \%$ of the peaks were used in order to best extract the mean of the summits.

In addition to the surface topography images, contact area images were also acquired using confocal microscopy. The instrument has the ability to collect high-resolution images exclusively from a single focal plane. This is beneficial because contact occurs within a thin layer at the padwafer interface. ${ }^{(8,9)}$ In order to acquire the contact area images, a specialized sample stage was used to apply a known downforce to the back of the pad sample. The polished side of the pad sample was placed facing down against a sapphire window. The known surface area of the pad sample/sapphire window contact was used to apply the appropriate downforce in order to create 4 PSI applied pressure. As sapphire has a similar refractive index compared to that of a polyurethane pad, it is suitable to simulate the pad-wafer contact during CMP. ${ }^{(14)}$ Since the two values of refractive index are similar, the lowest reflection occurs at the contact points. Light incident on a contact region is therefore not reflected back, producing a black area in the reflected image. Noncontact areas produce a reflected image because of discontinuities in the refraction index at the sapphire-air-pad interfaces. As with the topographic image acquisition, eight contiguous and nonoverlapping $425 \times 425 \mu^{2}$ images were taken in approximately the same location as the topographic images. These eight images were then stitched together to form one image covering a pad land area of 3,400 $\times 425 \mu \mathrm{m}^{2}$. Each stitched image contains 8,192 × 1,024 pixels therefore the 
image resolution is $0.42 \mu \mathrm{m} / \mathrm{pixel}$. Contact area, in $\mathrm{mm}^{2}$, and the number of points of contact was extracted from each of the stitched $3400 \times 425 \mu \mathrm{m}^{2}$ images. Contact area percentage and contact density were then calculated through the division of both the contact area and the number of points of contact by the total analyzed area in $\mathrm{mm}^{2}$, respectively. The detailed description of the confocal microscopy configuration and experimental setup is provided elsewhere. ${ }^{(8,9)}$

\section{Results and Discussion}

Pad surface topography images acquired from confocal microscopy are shown in Figure 1. In order to emphasize the high and low areas of the topography images, a transformation was performed by taking the inverse hyperbolic sine of the height. This creates a better visualization of the pad surface topography. ${ }^{(15)}$ The colors in Figure 1 represent different values of the surface height. The blue color is the lowest part of the pad surface topography, particularly the dark blue color. Blue colors are therefore representative of its pores. The green in Figure 1 is the mean of the surface topography, which is therefore representative of the interpore areas with a height in between that of the pores and the asperities. The orange, and in particular, the red in Figure 1 are the highest areas of the pad surface topography and are therefore representative of its asperities. During breakin the conditioner has the highest chance of coming into contact with the highest regions of the pad surface. This means that the conditioner will have more of an effect on pad asperities than the pores. This is evident from Figure 1 because the blue areas (pores) do not become more or less prominent throughout an hour of break-in at either magnitude of downforce. However, a change in the prominence of the orange and red areas over time in Figure 1 can be observed in all cases. This description of the topography images from Figure 1 indicates that the pad conditioned with a higher downforce is able to reach a stable topography within the first 5 to 15 minutes, while the 
pad conditioned at a lower downforce does not reach a completely stable value of surface topography until 60 minutes. This is evidenced by changes in surface height that can be seen on the pad conditioned at a higher downforce. After the first 5 minutes of conditioning at $10 \mathrm{lb} f$ the pad surface height changes dramatically, as can be seen by the decrease in the number of high summits from 0 to 5 minutes. ${ }^{(15)}$ However, it can be seen that the pad broken-in at $6 \mathrm{lb}_{\mathrm{f}}$ is subjected to a more gradual change in pad summit height, and never reaches the extent of the color change that happens to the pad broken-in at $10 \mathrm{lb}_{\mathrm{f}}$. While these observations are evident from the topographic images, however, such visual observation is not thorough enough. The topographic data needs to be compared in a more quantitative way. This can be done through analysis of the PDF, mean summit height, and mean summit curvature.

The pad surface height PDFs as shown in Figure 3 were generated from the respective topographic images. ${ }^{(8-10)}$ The left-hand tail of the PDF is produced from the lower areas of the pad surface. This means that the left-hand tail of the PDF is representative of the pores shown in blue in the topographic images from Figure 1. The left-hand tail of the PDF remains consistent throughout the pad-break in process indicating that pores remained stable throughout. This is expected because the conditioner has less of a chance of reaching the lower features of the pad surface. The mean of the PDF is adjusted to zero and is approximately representative of the green color shown in the topographic images. The right-hand tail of the PDF curve represents the uppermost surface of the pad, shown in orange and red in the topographic images, which can also be thought of as the summits of the pad asperities. ${ }^{(10)}$ This is therefore important because the summits of the pad asperities are the highest parts of the pad surface and are most likely to come into contact with the wafer during polishing. ${ }^{(2,3)}$ The conditioning disc cuts the higher pad features as the pad break-in progresses. As a result, the right-hand tails of the PDF curves at each conditioning downforce shift 
to the left and begin to align as the break-in time increases. As expected, the right-hand tail of the PDF curves for the pad broken-in at the higher downforce aligns faster to the left compared to the pad broken-in at the lower downforce. Such trends are justifiable as higher pad conditioning downforce is expected to cut high pad features faster than lower conditioning downforce. Further observance of the right-hand tail of the PDF of the pad conditioned at $10 \mathrm{lb}$ shows that the righthand tails of the different break-in times begin to align at 15 minutes, and almost completely overlap at 30 and 60 minutes. This alignment, and eventual overlap, of the right-hand tail of the PDF is evidence that pad break-in for longer than 5 to 15 minutes at a comparatively high downforce $(10 \mathrm{lb} f)$ leads to progressive surface flattening. ${ }^{(15)}$

The topographic images were also used to generate values of mean summit height and mean summit curvature at each pad break-in time for the two different downforces. ${ }^{(8,9)}$ These generated values were plotted against break-in time in Figure 3. Generally, as break-in time increases, mean summit height decreases (pad asperities become shorter) and mean summit curvature becomes smaller (asperity tips become flatter). This is to be expected because the goal of the conditioner is cut the upper most asperities of the pad surface until a more uniform pad surface is created. ${ }^{(2,3)}$ During the first 30 minutes of break-in in, the mean summit height shown in Figure 3, appears to asymptotically approach different values for each downforce but, given enough break-in time, the mean summit height at $6 \mathrm{lbf}$ begins to converge towards the mean summit height for $10 \mathrm{lb}$. It is expected that the mean summit height will begin to become more stable as break-in time increases because the asperities of the pad will be cut and deformed plastically, until the force applied to the asperities drops below the threshold for plastic deformation and they begin to behave elastically. This means that, as the conditioner either cuts away or plastically deforms the uppermost asperities, it will gradually come into contact with more asperities that were previously too short 
to come into contact with the conditioner. As the conditioner comes into contact with more asperities, the force that the conditioner exerts on each individual asperity will become smaller, until such force on each individual asperity is not enough to cause plastic deformation and cutting. At this threshold, the asperities begin to behave elastically and the pad surface has reached a more stable mean summit height. At both conditioning downforces, the mean summit curvature increases in the first 5 minutes and proceeds to reach a lower value as break-in progresses. With longer pad break-in time, the conditioner shaves away the sharpness of the asperities and stable values of mean summit curvature can be attained after 15 minutes. While we are not sure regarding the actual mechanism as to why pad break-in initially causes sharper pad asperities during the early break-in time before it reaches lower and more stable values, such a phenomenon emphasizes the importance of reasonable pad break-in time prior to beginning polishing processes. As with the mean summit height, the values of mean summit curvature of the pads broken-in at different downforces appear to be converging given enough pad break-in time.

The contact area images shown in Figure 4 are displayed in the same manner as those in Figure 1. Comparison of the contact images at the two downforces shows that the pad broken-in at the higher conditioning downforces results in larger contact areas as shown by the larger black spots. ${ }^{(8,9)}$ These large contact areas are more apparent at a higher conditioning downforce, especially after 30 and 60 minutes of pad break-in. Further analysis of Figs. 1 and 4 is presented in Figure 5 to better explain the mechanics of the large contacts being generated at a conditioner downforce of $10 \mathrm{lb}$. Figure 5 (left) and (middle) are extracted from the first topographic and contact image of Figs. 1 and 4 after 60 minutes of pad break-in. Those images are then compared to a summit contour plot in Figure 5 (right). ${ }^{(8,9)}$ It must be emphasized that all three images are exactly from the same spot on the pad. Figure 5 (right) shows the summit contours at $25 \%$ of the maximum pad 
surface height. Each red dot represents the location of the maximum summit at the top of each summit contour. While each red dot represents the highest point at a given summit contour, however, it may not be high enough as compared to the other red dots to result in pad-wafer contact. Based on Figure 5, there are three possible types of contacting summits as illustrated in Figure 6. An ideal, fully supported, summit with solid underlying pad material is illustrated on the left in Figure 6 and shown in Figure 5 using an arrow designated as (i). We know this to be a fully supported summit because of the large area within the top $25 \%$ contour, which is associated with the very small amount of black contact and a relatively large distance from any pores. ${ }^{(16)} \mathrm{A}$ fractured, poorly supported contacting summit is illustrated on the right in Figure 6 and shown in Figure 5 using an arrow designated as (iii). The illustration in the middle of Figure 6 is a partially supported contacting summit and can be thought of as something in-between a fully supported and poorly supported contacting summit. This type of contact is shown in Figure 5 using an arrow designated as (ii). By observing the contact and topographic images, we can tell that the fully supported contacting summit is uncommon while a a summit that is either partially or poorly supported is much more prevalent ${ }^{(16)}$ These partially and poorly supported contacting summits can be seen throughout the contact and topographic images in Figs. 1 and 4, and are mostly associated with the edge of pores. This indicates that many contacting summits are generated from broken or unsupported pore walls and it is possible for these summits to partially or fully cover pores as shown in Figure 5(iii). Applying a force to a partially or poorly supported summit would produce a large, low pressure contact area. ${ }^{(16)}$ This would hold true particularly when the partially or poorly supported summit took the form of the large pad fragment shown at the top of the summit on the right in Figs. 6 and Figure 5 using the arrow designated as (iii). 
From the contact images in Figure 4, the mean contact density and percentage contact area at each break-in time are calculated and reported in Figure $7 \cdot^{(8,9)}$ It should be noted that measurable variabilities in the contact area and contact density throughout the pad break-in process should be expected. Different pad samples were extracted during break-in for surface topography analysis. As such, the pad surface will therefore have slight variability from sample to sample and location to location. This can cause measurable variabilities in the results which will be amplified especially when the pad surface has not yet reached steady-state conditions. An example of this amplification of variability can be seen during the 5 to 15 minute time period in the case of the contact area at $10 \mathrm{lb} f$ in Figure 7. This large variation provides further evidence that the pad has not yet stabilized during the 5 to 15 minute time period. Furthermore, the contact density and percentage contact area curves at $6 \mathrm{lb}_{\mathrm{f}}$ continue to have more fluctuations throughout the pad break-in process compared to the pad broken-in at $10 \mathrm{lb}$. This provides further evidence that break-in at the lower downforce does not create as stable of a pad surface micro-texture as that at the higher downforce in the same time range. This level of variability does not affect the analysis of the results as the overall trends can still be observed and compared. For this reason, trend lines (i.e. a dashed-line and solid line curves) have been fitted through the $6 \mathrm{lb}_{\mathrm{f}}$ and $10 \mathrm{lb}_{\mathrm{f}}$ curves, respectively. Results from Figure 7 do not differentiate the three types of contacting summits and show that a higher conditioning downforce generally results in a greater contact area and contact density. This clearly indicates that the higher conditioning downforce generates faster and higher amounts of pad fragments and, possibly, fractured or collapsed pore walls than a lower conditioning downforce. Also, contrary to mean summit height and mean summit curvature both contact parameters reach a stable value within the first 15 to 30 minutes of break-in regardless of downforce. 
Various surface micro-texture parameters will reach a stable value within different time ranges. At both values of downforce, contact density and percentage contact area become stable after approximately 30 minutes, while it takes 60 minutes for mean summit height and mean summit curvature to become stable at 6 lbf. Since a pad surface cannot be considered to be stable until all of the relevant parameters have leveled off, we can say that the pad conditioned at $6 \mathrm{lb}_{\mathrm{f}}$ has not reached stability until approximately 60 minutes or later, while the pad conditioned at $10 \mathrm{lb}_{\mathrm{f}}$ has stabilized in just 30 minutes. Assuming that, each of downforce, all micro-texture parameters have become stable within 60 minutes, Table I can be used for easy comparison of stable values extracted at each downforce. The values of contact density and percentage contact area are in agreement with prior results reported by our research group. ${ }^{(5-10)}$ The values for mean summit height and mean summit curvature are similar for both magnitudes of downforce, but the values of contact density and percentage contact area are twice as large for the pad broken-in at the higher downforce. This further indicates that given enough break-in time, mean summit height and curvature will reach the same stable value, while contact density and percentage contact area will not.

\section{Conclusion}

This study investigated the effect of conditioning downforce on the evolution of the micro-texture of the pad surface during break-in. Two different downforces were used, each resulting in different evolution paths during break-in. Throughout break-in, pad samples were collected and their surface topography and contact area analyzed with confocal microscopy. The pad conditioned at the lower downforce resulted in a smaller percentage contact area and a smaller contact density. 
This held true once the pad surface micro-texture reached stable values of contact parameters for each downforce. The pad conditioned at the lower downforce also had taller and sharper asperities throughout the break-in process, but both appeared to approach the same stable surface topography values for each downforce after 60 minutes. The pad conditioned at the higher downforce generated more pad fragments, which after analysis were shown to be large, low pressure contact areas that would contribute to a higher lubrication during polishing. These were associated with poorly supported contacting summits, which were shown to be much more common than conventional fully supported contacting summits. The pad conditioned at the higher downforce was able to reach a stable value of mean summit height and mean summit curvature within the first 5 to 15 minutes of the break-in process. It was able to reach a stable value of contact density and percentage contact area within the first 15 to 30 minutes of the break-in process. The pad conditioned at the lower downforce was not able to reach a completely stable value of mean summit height and mean summit curvature within the first 60 minutes, while it was able to reach a stable value of contact density and percentage contact area within the first 15 to 30 minutes of the break-in process. Even though the pad conditioned at the lower downforce did not fully reach a stable value of mean summit height and mean summit curvature it began to approach a stable value after approximately 60 minutes. Both downforces were compared at a break-in time of 60 minutes to ensure that both pad surface micro-textures were stabilized and had approximately reached steady-state. This comparison resulted in values of contact area and contact density that were twice as large at the higher downforce. We think that conducting this experiment with different conditioners will be beneficial for developing a better understanding of the role downforce has on the evolution of the micro-texture of the surface of the pad during break-in. We hope that this will help users in screening different types of discs and different break-in times. 


\section{References}

1. S. Wolf. Microchip Manufacturing. Lattice Press, 2004.

2. Y. Li. Microelectronic Applications of Chemical Mechanical Planarization. John Wiley \& Sons, 2007.

3. J. Steigerwald, S. Murarka and R. Gutmann. Chemical Mechanical Planarization of Microelectronic Materials. John Wiley \& Sons, 2008.

4. T. Sun, L. Borucki, Y. Zhuang and A. Philipossian, Microelectronic Engineering, 87, 553 (2009).

5. Y. Sampurno, A. Rice, Y. Zhuang and A. Philipossian, Electrochemical and Solid-State Letters, 14, 318 (2011).

6. Y. Mu, R. Han, Y. Sampurno, Y. Zhuang, and A. Philipossian, ECS J. Solid State Sci. Tehcnol., 5,6 345 (2016).

7. B. Vasilev, S. Bott, R. Rzehak and J. Bartha, Microelectronic Engineering, 111, 21 (2013).

8. T. Sun, L. Borucki, Y. Zhuang, Y. Sampurno, F. Sudargho, X. Wei, S. Anjur and A. Philipossian, Jpn. J. Appl. Phys., 49, 026501 (2010).

9. T. Sun, Y. Zhuang, L. Borucki and A. Philipossian, Jpn. J. Appl. Phys., 49, 046501 (2010).

10. R. Han, Y. Mu, Y. Sampurno, Y. Zhuang and A. Philipossian, ECS J. Solid State Sci. Tehcnol., 6, 201 (2017).

11. X. Liao, Y. Zhuang, L. Borucki, J. Cheng, S. Theng, T. Ashizawa and A. Philipossian, Jpn. J. Appl. Phys., 52, 018001 (2013).

12. H. Jeong, K. Park and K. Cho, Annals of the CIRP., 56, 357 (2007).

13. http://aracainc.com/equipment/r-and-d-polishers/

14. C. Elmufdi and G. Muldowney, Mater. Res. Soc. Symp. Proc., 914, F12-06 (2006). 
15. C. Stuffle, R. Han, Y. Sampurno, D. Slutz, S. Theng, L, Borucki and A. Philipossian, ECS J. Solid State Sci. Technol., 7, 9 (2018).

16. L. Borucki, Y. Sampurno, Y. Zhuang, and A. Philipossian :The Origin and Mechanics of Large Pad-Wafer Contact Areas. In: Proceedings of 14th International Symposium on Chemical Mechanical Planarization, Lake Placid, NY USA (2009). 
Table I. Pad surface micro-texture parameters after 60 minutes of pad break-in.

\begin{tabular}{|c|c|c|c|c|}
\hline $\begin{array}{c}\text { Conditioning } \\
\text { Downforce } \\
\left(\mathrm{lb}_{\mathrm{f}}\right)\end{array}$ & $\begin{array}{l}\text { Mean Summit Height } \\
(\mu \mathrm{m})\end{array}$ & $\begin{array}{l}\text { Mean Summit Curvature } \\
\qquad\left(1 / \mu \mathrm{m}^{2}\right)\end{array}$ & $\begin{array}{c}\text { Contact Density } \\
\text { (contact count per mm²) }\end{array}$ & $\begin{array}{c}\text { Percentage Contact Area } \\
\text { (\%) }\end{array}$ \\
\hline 6 & 21 & 407 & 82 & 0.022 \\
\hline 10 & 20.5 & 323 & 143 & 0.052 \\
\hline
\end{tabular}



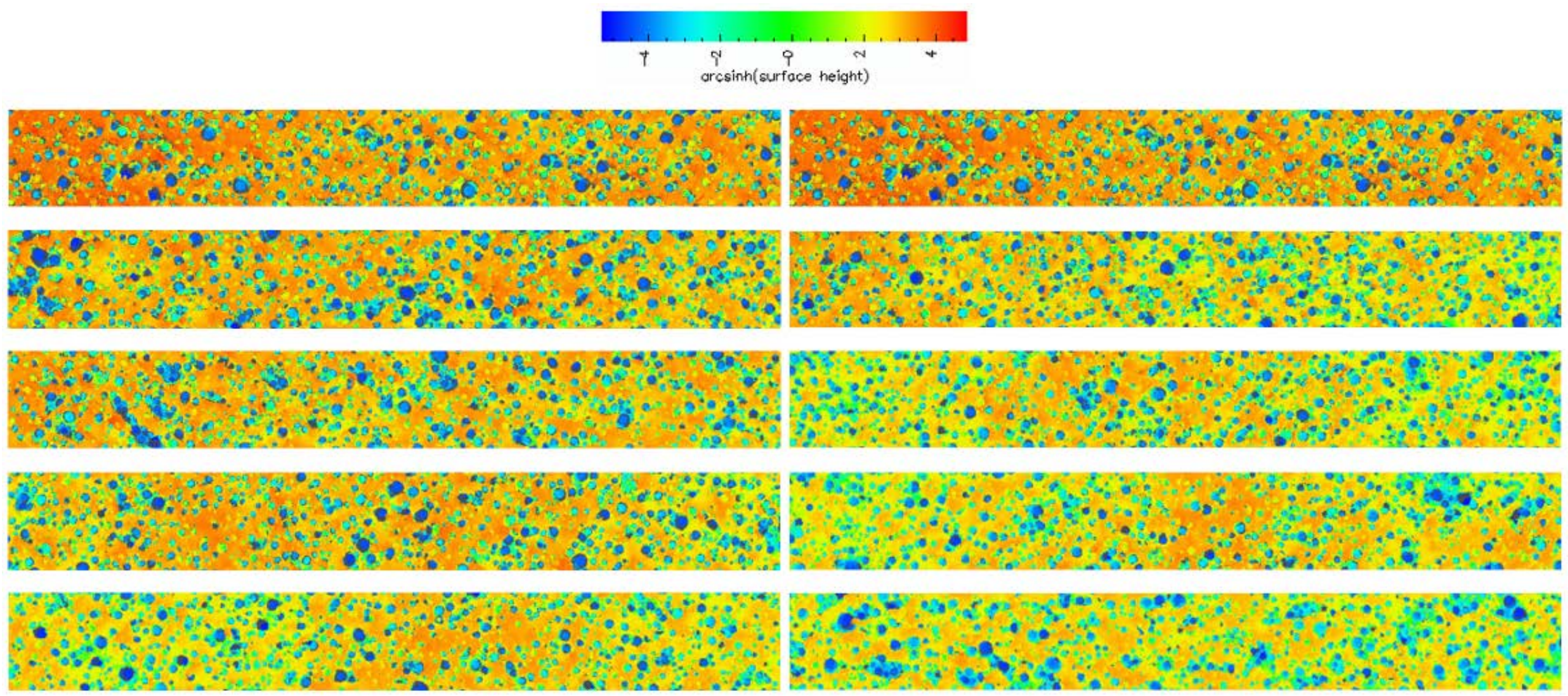

Figure 1: Surface topography of pads broken-in with downforce of $6 \mathrm{lb} \mathrm{b}_{f}$ (left) and $10 \mathrm{lb}$ (right) prior to break-in (top) and at 5, 15, 30, and 60 minutes (bottom) after pad break-in. 

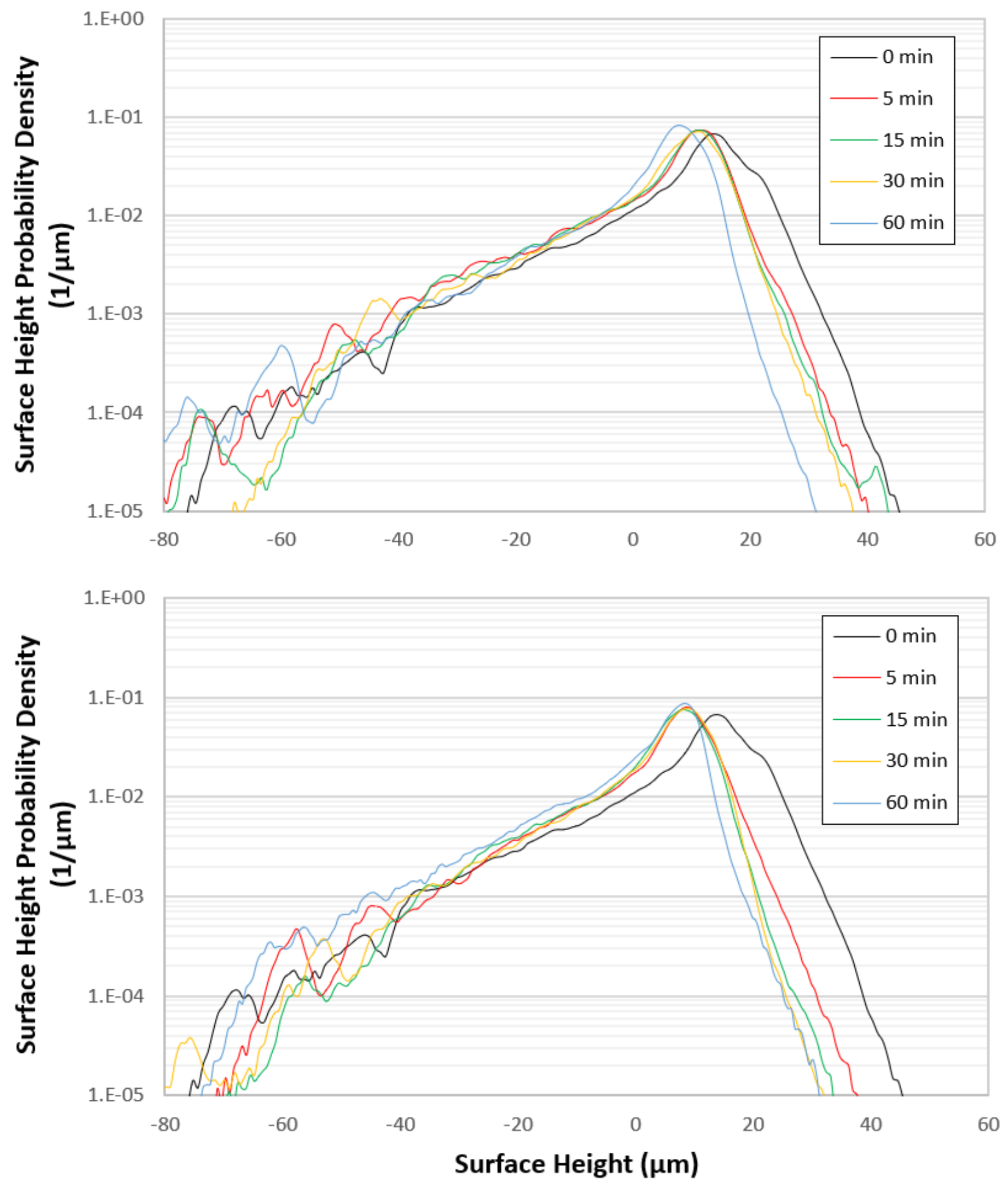

Figure 2: Surface height probability density functions of pads broken-in with downforce of $6 \mathrm{lb}_{f}(\mathrm{top})$ and $10 \mathrm{lb}_{f}$ (bottom) prior to break in (0 minutes) and at 5, 15, 30, and 60 minutes after pad break-in. 

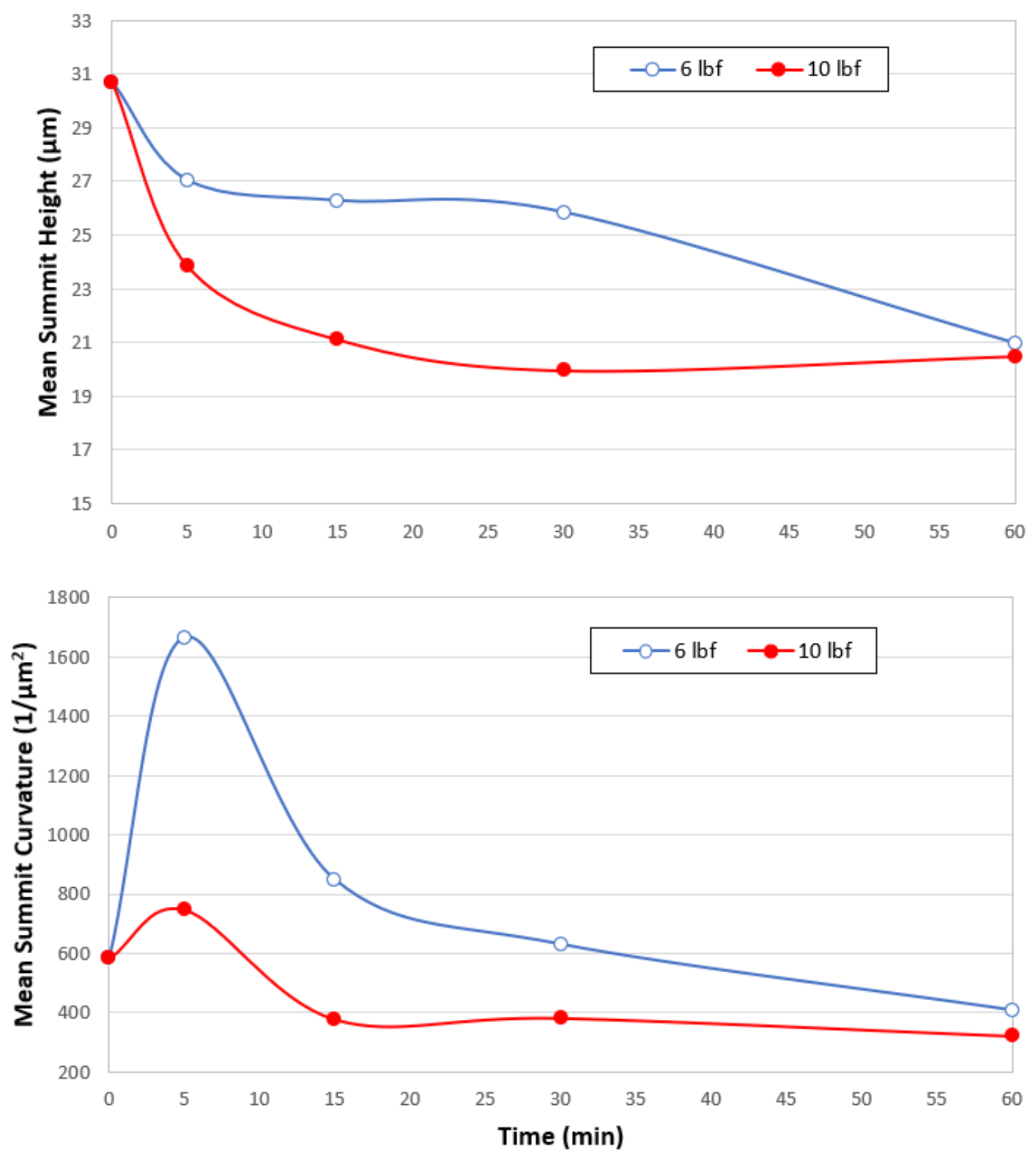

Figure 3: Mean Summit Height and Mean Summit Curvature vs. break-in time. 

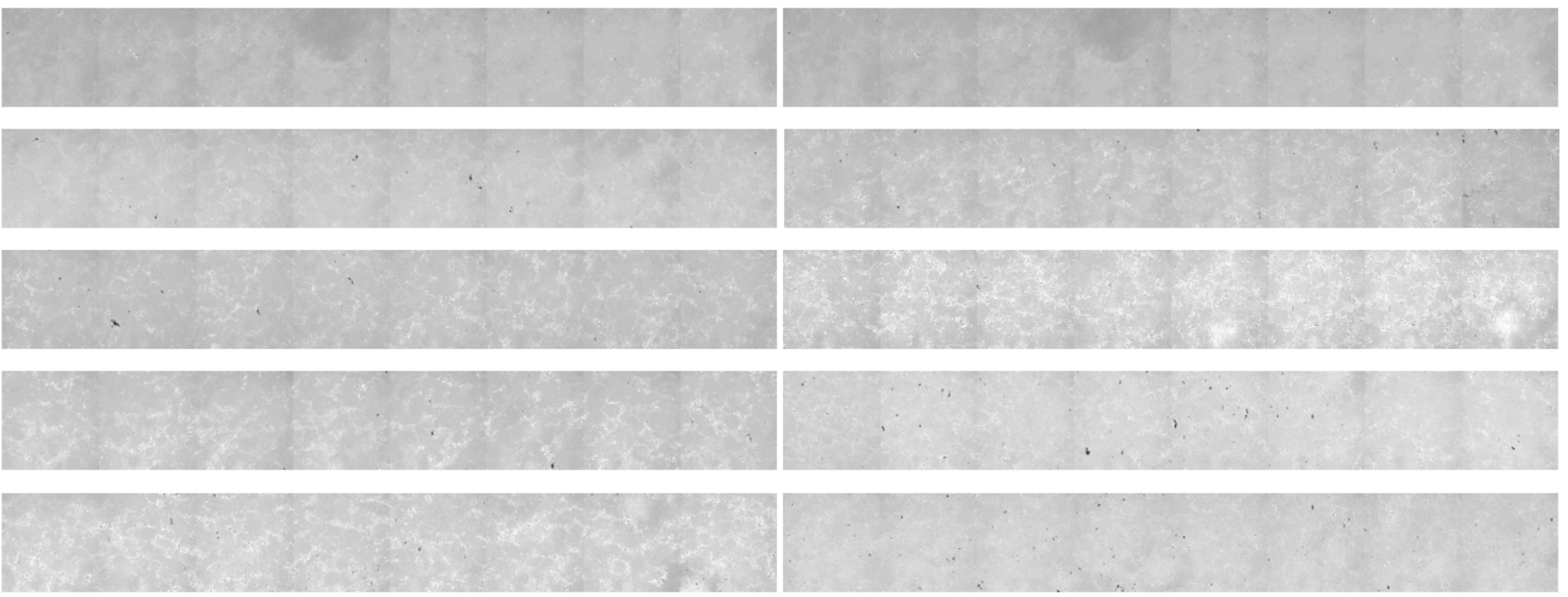

Figure 4: Contact images of pads broken-in with conditioning downforce of $6 \mathrm{lb}$ (left) and $10 \mathrm{lb}$ (right) at initial (top) and 5, 15, 30, and 60 minutes (bottom) after pad break-in. 


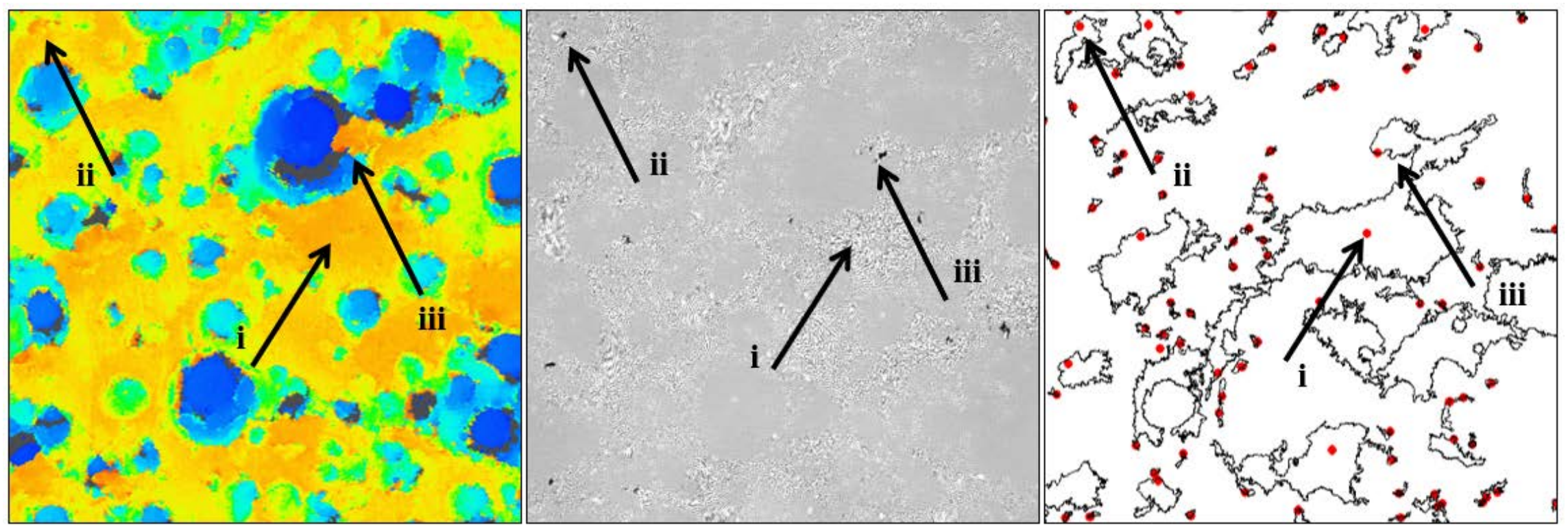

Figure 5: Three types of contact. Topographic image (left), contact image (middle), and a summit contour plot (right). 


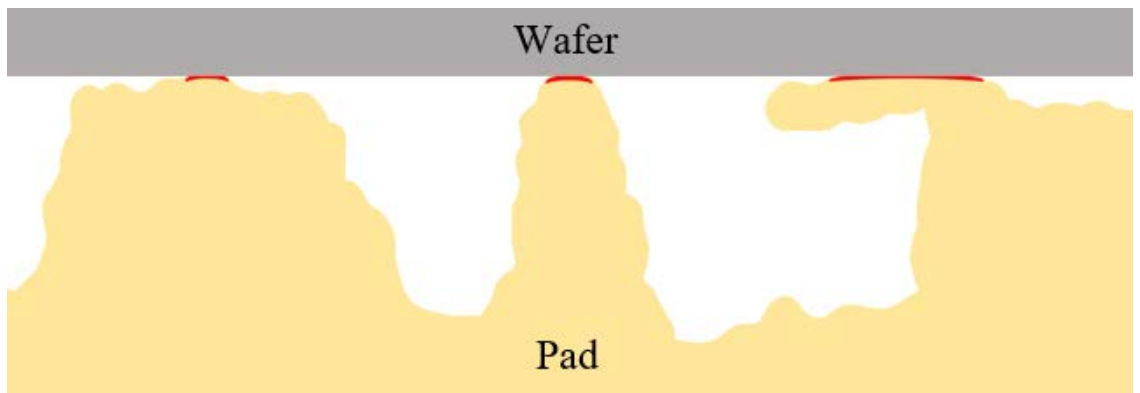

Figure 6: Three types of asperity contact (highlighted in red). Fully supported (left), partially supported (middle), and poorly supported (right). 

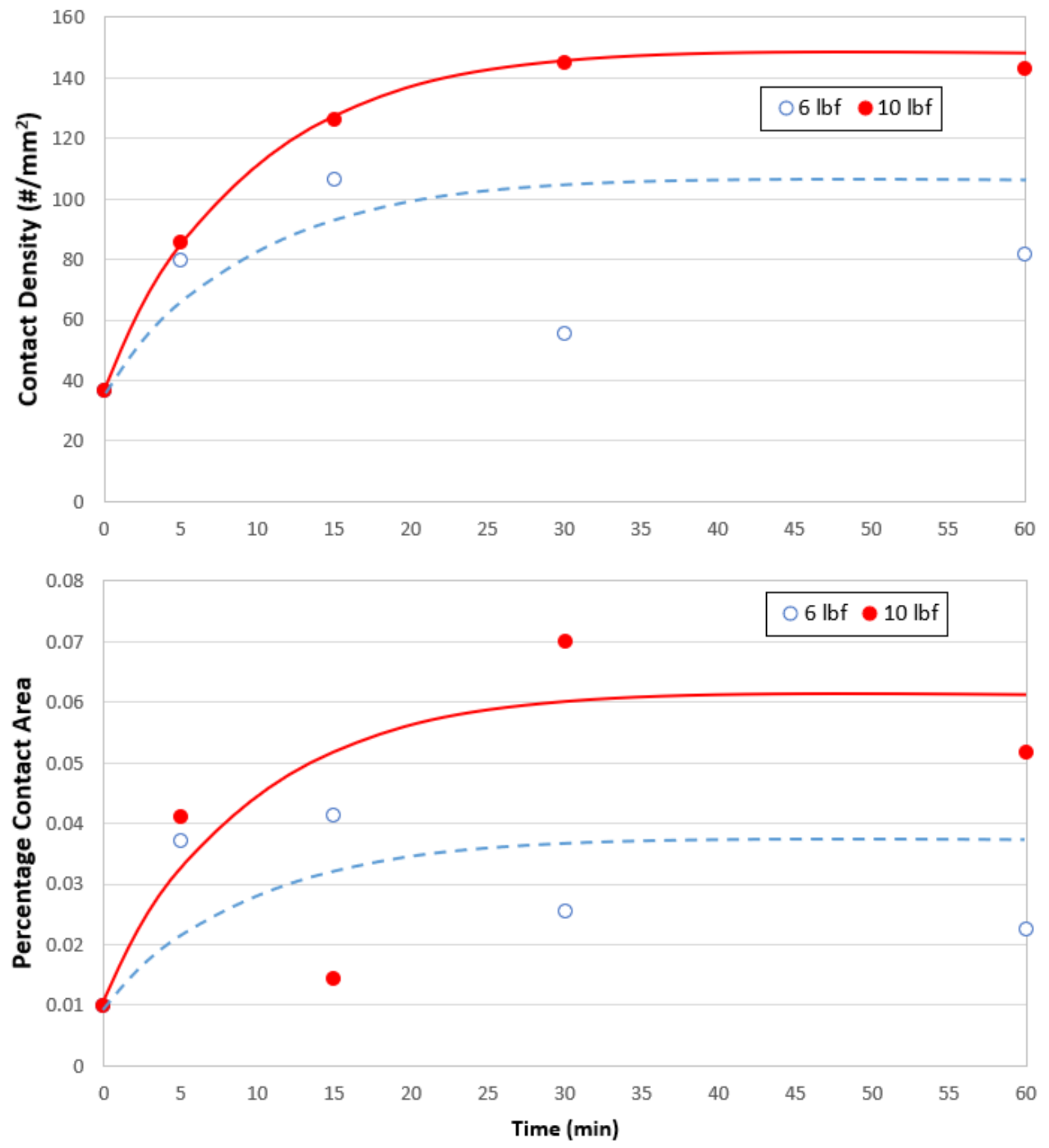

Figure 7: Contact Density and Percentage Contact Area vs. break-in time. 